\title{
Impaired blood rheology is associated with endothelial dysfunction in patients with coronary risk factors
}

\author{
Hideki Yagi, Hiroyuki Sumino*, Tomoyuki Aoki, Katsuhiko Tsunekawa, Osamu Araki, \\ Takao Kimura, Makoto Nara, Takayuki Ogiwara and Masami Murakami \\ Department of Clinical Laboratory Medicine, Gunma University Graduate School of Medicine, \\ Maebashi, Japan
}

\begin{abstract}
To investigate the relationship between blood rheology and endothelial function in patients with coronary risk factors, brachial arterial flow-mediated vasodilatation (FMD), an index of endothelial function and blood passage time (BPT), an index of blood rheology, and fasting blood cell count, glucose metabolism, and plasma fibrinogen, lipid, C-reactive protein, and whole blood viscosity levels were measured in 95 patients with coronary risk factors and 37 healthy controls. Brachial arterial FMD after reactive hyperemia was assessed by ultrasonography. BPT was assessed using the microchannel method. In healthy controls, BPT significantly correlated with FMD $(r=-0.325, p<0.05)$, HDL cholesterol $(r=-0.393, p<0.05)$, body mass index (BMI; $r=0.530, p<0.01)$, and plasma fibrinogen concentration $(r=0.335, p<0.05)$. In a multivariate regression analysis adjusted for all clinical variables, BPT remained significantly associated with BMI and fibrinogen, but not with FMD, in healthy controls. In patients with coronary risk factors, BPT significantly correlated with FMD $(r=-0.331$, $p<0.01)$, HDL cholesterol $(r=-0.241, p<0.05)$, BMI $(r=0.290, p<0.01)$, hematocrit $(r=0.422, p<0.001)$, white blood cell count $(r=0.295, p<0.01)$, platelet count $(r=0.204, p<0.05)$, and insulin $(r=0.210, p<0.05)$. In a multivariate regression analysis adjusted for all clinical variables, BPT remained strongly associated with FMD and hematocrit in patients with coronary risk factors. These data indicate that BPT is closely associated with FMD in patients with coronary risk factors and suggest that the measurement of blood rheology using the microchannel method may be useful in evaluating brachial arterial endothelial function as a marker of atherosclerosis in these patients.
\end{abstract}

Keywords: Blood rheology, coronary risk factors, endothelial dysfunction, patients

\section{Introduction}

Epidemiological studies have identified hypertension [6, 17], dyslipidemia [6, 16], diabetes mellitus [18], and smoking [6, 49] as important risk factors for coronary heart disease (CHD). These coronary risk factors induce and promote atherogenesis [38]. Recent insights into the basic mechanisms involved in atherogenesis indicate that deleterious alterations of endothelial physiology, also termed endothelial dysfunction, represent a key early step in the development of atherosclerosis and are also involved in plaque progression and occurrence of atherosclerotic complications [38]. In the early stages of atherosclerosis, these coronary risk factors are possible causes of endothelial dysfunction [38].

Despite recent advances in our understanding of the pathogenesis of atherosclerosis, the pathophysiology of the coronary risk factor-associated atherosclerotic process is poorly understood. Hemorheological parameters are considered related to the formation of atherosclerotic thrombi because

\footnotetext{
*Corresponding author: Hiroyuki Sumino, MD, Department of Clinical Laboratory Medicine, Gunma University Graduate School of Medicine, 3-39-15 Showa-machi, Maebashi, Gunma 371-8511, Japan. Tel.: +81 27220 8576; Fax: +81 27220 8583; E-mail: suminoh@gunma-u.ac.jp.
} 
fibrinogen concentration [9], plasma viscosity [23], and blood viscosity [28] have been identified as independent atherosclerotic risk factors. Several clinical studies have reported a relationship between hemorheological parameters and coronary risk factors [12, 22, 25-27]. Hemostatic factors, including fibrinogen level, whole-blood viscosity, plasma viscosity, impaired erythrocyte deformability, and platelet aggregation, are greater in hypertensive patients than those in normotensive individuals [25, 27]. Dyslipidemic patients were found to exhibit high serum levels of total cholesterol (TC), lowdensity lipoprotein cholesterol (LDL-C), and triglyceride (TG), which impaired the deformability of erythrocytes [26]. Plasma viscosity positively correlates with TC, TG, and LDL-C levels and inversely correlates with high-density lipoprotein cholesterol (HDL-C) level [22]. Smokers exhibit higher wholeblood viscosity, plasma viscosity, and plasma fibrinogen concentrations compared with nonsmokers [12].

Recently, a new microchannel method has been developed to measure blood rheology [19, 29, 30, 32 , $33,42,43,45,46]$. The method facilitates the observation of blood flow under a microscope connected to a visual display unit while evaluating blood rheology. This method may be useful in acquiring new insights into the pathophysiology of the atherosclerotic process in patients with coronary risk factors. Reportedly, blood rheology measured using the microchannel method is influenced by red blood cell (RBC) deformability, leucocyte adhesiveness, platelet aggregation, and whole-blood and plasma viscosities $[19,29,30,32,33,42,43,45,46]$. Using the microchannel method, several studies have identified a relationship between blood rheology and coronary risk factors [29, 33, 42, 43, 46]. Blood rheology is impaired in patients with hypertension [46] and dyslipidemia [29, 33] and smokers [43] and is positively correlated with TC, TG, and LDL-C levels and the LDL-C/HDL-C ratio and negatively correlated with HDL-C [29, 33, 42]. However, the relationship between blood rheology and endothelial function in patients with coronary risk factors remains unclear.

Brachial arterial flow-mediated vasodilatation (FMD) is dependent on endothelial function and can be measured during reactive hyperemia using high-resolution ultrasound [3, 37]. FMD is widely used in clinical settings because it serves as a good marker of clinical atherosclerosis [3, 37]. Impaired brachial arterial FMD is associated with cardiovascular disease. To investigate the relationship between blood rheology and endothelial function in patients with coronary risk factors, we compared brachial arterial FMD and blood rheology between healthy individuals and patients with coronary risk factors. In addition, we evaluated the correlation between brachial arterial FMD and blood rheology in healthy individuals and patients with coronary risk factors.

\section{Materials and methods}

\subsection{Participants}

We recruited 132 consecutive Japanese volunteers (age: $48.9 \pm 13.2$ years; range: $22-74$ years), consisting of 72 men (age: $49.9 \pm 13.6$ years; range: $22-74$ years) and 60 women (age: $47.7 \pm 12.7$ years; range: $23-68$ years). The participants were referred to our department to evaluate the risk or presence of cardiovascular disease. All were in a stable chronic condition. Details of patients' medical histories were acquired, and physical examinations, examinations of brachial arterial endothelial function, rheological measurement of whole blood, and laboratory tests were performed for all participants. Patients with a history of cardiovascular disease, including stroke, CHD, thromboembolic disease, congestive heart failure or peripheral arterial disease, malignancy, infectious disease, liver disease, renal disease, overt endocrine disease, or use of steroid hormones, nitrates, antiplatelets, or anticoagulants, were excluded because these conditions may have a serious influence on both endothelial function and rheological measurement. 
The participants were divided into two groups: the healthy control group $(n=37)$ and the patients with coronary risk factors group $(n=95)$. Sixty-seven percent of the patients with coronary risk factors had one or more classical coronary risk factor, such as hypertension, dyslipidemia, diabetes mellitus, or current smoking. Hypertension, dyslipidemia, and diabetes mellitus were defined according to diagnostic criteria $[1,35,47]$ or if participants were receiving any medications for these diseases. Of the patients with coronary risk factors, patients with hypertension, dyslipidemia, diabetes, and current smokers comprised $48 \%, 79 \%, 14 \%$, and 29\%, respectively, of the cohort. Patients with hypertension were treated as follows: $9 \%$ with angiotensin II type 1 receptor blockers, $2 \%$ with angiotensin-converting enzyme inhibitors, $1 \%$ with $\alpha 1$-blockers, $3 \%$ with $\beta$-blockers, $13 \%$ with calcium antagonists, and $5 \%$ with diuretics. Patients with dyslipidemia were treated as follows: $9 \%$ with statins, $1 \%$ with ezetimibes, and $1 \%$ with fibrates. Patients with diabetes were treated as follows: $3 \%$ with dipeptidyl peptidase- 4 inhibitors, $2 \%$ with sulfonylureas, and $1 \%$ with insulin therapy. Written informed consent was obtained from each participant, and the study was approved by the Institutional Review Board of Gunma University Hospital, Gunma, Japan.

\subsection{Study protocol}

In the morning, after a 12-h fast, physical examinations and examinations of brachial arterial endothelial function were performed, and blood samples were collected in three polypropylene tubes for serum and plasma analyses and blood rheology and blood viscosity measurements. Blood rheology and blood viscosity samples (each $2 \mathrm{~mL}$ ) were obtained by puncture of an antecubital vein using a 23-G needle whilst the patient was in the sitting position. Heparin solution $(0.1 \mathrm{~mL}, 1000 \mathrm{IU} / \mathrm{mL})$ and EDTA-2K were used as anticoagulants for measurement of whole blood rheology and whole blood viscosity, respectively. The rheology of whole-blood samples was measured within $2 \mathrm{~h}$ of sample collection. The viscosity of whole-blood samples was measured immediately after sample collection.

\subsection{Physical examination}

The height and weight of each participant were measured, and their body mass index (BMI) was calculated (weight in kilograms divided by height in meters squared). Blood pressure was measured in the morning after overnight fasting $(12 \mathrm{~h})$. Measurements were conducted by the same investigator with a sphygmomanometer on the right arm of the patients, after he or she had rested for $10 \mathrm{~min}$ in the supine position.

\subsection{Measurement of blood rheology}

Blood rheology was measured with a microchannel array flow analyzer (MC-FAN; Hitachi Haramachi Electronics Co., Ltd., Ibaraki, Japan), as previously reported [29, 30, 32, 33, 42, 43, 45, 46]. Briefly, an aliquot $(200 \mu \mathrm{L})$ of each blood sample was introduced into a cylinder connected to the inlet hole of the silicon chip holder using a 1-mL disposable syringe and a thin catheter. The blood sample was allowed to flow through the microchannel array (Bloody 6-7; Hitachi Haramachi Electronics Co., Ltd.; V-shaped groove; width $7 \mu \mathrm{m}$, length $30 \mu \mathrm{m}$, depth $4.5 \mu \mathrm{m}$ ) by applying a pressure difference of $20 \mathrm{~cm}$ of water. The flow rate was determined by recording the time when the meniscus of the sample crossed the graduation marks $(10 \mu \mathrm{L}$ intervals from $0-100 \mu \mathrm{L})$ on the sample cylinder. Simultaneously, the flow of blood cells through individual microchannels was observed and recorded using an inverted metallographic microscope, video camera, and video recorder. The passage time of $100 \mu \mathrm{L}$ of saline was determined before each blood measurement to check the accuracy of the equipment ( $12 \mathrm{sec}$; permissible range: $10-14 \mathrm{sec}$ ), and this information was then used to correct the passage time of $100 \mu \mathrm{L}$ 
of whole blood. The corrected passage time of whole blood was calculated as (observed passage time of whole blood $\times 12$ )/observed passage time of saline, and it was designated blood passage time (BPT). The inter- and intra-assay coefficients of variation for BPT were $8 \%$ and 5\%, respectively.

\subsection{Measurement of brachial arterial endothelial function}

The validity of this method has been demonstrated in previous studies [8, 44]. In brief, after participants had rested in the supine position for $10 \mathrm{~min}$, imaging of the right brachial artery and measurement of vasodilatory responses were conducted using high-resolution Doppler ultrasonography equipment with a 7.5-MHz transducer (LOGIQ 9; GE Healthcare, Japan Corporation, Tokyo, Japan). A nontortuous segment of the brachial artery was scanned longitudinally about $4-5 \mathrm{~cm}$ above the elbow, where the clearest images could be obtained. After determining the optimum transducer position, the skin was marked, and the arm was maintained in the same position throughout the study. After baseline images of the brachial artery were obtained and arterial flow velocity was determined, a blood pressure cuff tied around the proximal portion of the forearm, distal to the antecubital fossa, was inflated to $250 \mathrm{~mm} \mathrm{Hg}$ for $5 \mathrm{~min}$, followed by quick deflation. Flow velocity in the brachial artery was determined immediately and $1 \mathrm{~min}$ after deflation. All scans were recorded on videotape for later analysis along with blood pressure and heart rate recordings during each stage of the investigation. The diameter of the brachial artery was measured from the anterior to posterior interfaces between the media and adventitia (" $m$ " line) at the end of diastole, which was defined by the $\mathrm{R}$ wave on a continuously recorded electrocardiogram. The vessel diameter was measured over four cardiac cycles, and the measurements were averaged, without previous knowledge of the individual's treatment group. FMD was calculated as the percent increase in arterial diameter during hyperemia, and it represents an index of endotheliumdependent vasodilatation. The inter- and intra-observer variabilities for the repeated measurements of arterial diameter at rest were $0.06 \pm 0.03$ and $0.04 \pm 0.03 \mathrm{~mm}$, respectively. The variability of FMD determined on two separate days was $2.7 \% \pm 1.2 \%$.

\subsection{Laboratory analyses}

Hematocrit, white blood cell (WBC) count, and platelet count were measured using an automated hematology analyzer (Sysmex XE-5000; Sysmex Corporation, Kobe, Japan). Fibrinogen concentration was measured by the Clauss method using an automated coagulation analyzer (Sysmex CS-5100; Sysmex Corporation). Serum TC, TG, HDL-C, and LDL-C levels were measured by enzymatic methods, and $\mathrm{C}$-reactive protein (CRP) levels were measured by latex immunoassay, using an automatic analyzer (LABOSPECT008; Hitachi High-Technologies Corporation, Tokyo, Japan). Serum insulin levels were measured by chemiluminescence immunoassay using an automatic analyzer (AIA-2000 LA; Tosoh Corporation, Tokyo, Japan). Plasma glucose levels were measured by the hexokinase method, and hemoglobin A1c levels were measured by high-performance liquid chromatography, using automatic analyzers (ADAMS Glucose GA-1170 and ADAMS A1c HA-8180; ARKRAY, Inc., Kyoto, Japan). Whole blood viscosity levels were measured at 60 revolutions/min using a micro cone-plate viscometer (LVDV-II + Pro CP; Brookfield Engineering Laboratories, Middleboro, MA, USA) [36]. The interand intra-assay coefficients of variation for BPT were $7 \%$ and $5 \%$, respectively.

\subsection{Statistical analyses}

Data are expressed as means \pm standard deviation (SD). The Student's $t$-test was used to analyze differences between the two groups. The $\chi^{2}$ and Fisher's exact tests were used to compare the proportion of males to females and the prevalence of risk factors. Pearson's correlation coefficient was used 
to examine the relationships between BPT and FMD values and other clinical variables. Multiple regression analysis was performed between BPT and FMD values and other clinical variables. All probability values were two-tailed. $P$-values of $<0.05$ were considered statistically significant. All statistical analyses were performed using IBM SPSS software (v21.0; Michigan, IL, USA).

\section{Results}

\subsection{Participant characteristics}

The characteristics of healthy controls and patients with coronary risk factors are shown in Table 1 . Age, BMI, and systolic and diastolic blood pressures were significantly greater in patients with coronary risk factors than those in healthy controls (all $p<0.001$ ). BPT, hematocrit, WBC count, and fibrinogen, plasma glucose, hemoglobin A1c, insulin, TC, TG, LDL-C, CRP, and whole blood viscosity levels were also significantly greater in patients with coronary risk factors than those in healthy controls (all $p<0.05$ ), and brachial arterial FMD and HDL-C levels were significantly lower in patients with coronary risk factors than those in healthy controls (all $p<0.001)$. However, platelet count did not differ

Table 1

Characteristics of control participants and patients with coronary risk factors

\begin{tabular}{|c|c|c|c|}
\hline Variable & Control participants & Patients with coronary risk factors & $p$-value \\
\hline Number & 37 & 95 & \\
\hline Age (years) & $42.4 \pm 12.9$ & $51.4 \pm 12.5$ & $<0.001$ \\
\hline $\operatorname{Men}(n(\%))$ & $10(27)$ & $62(65)$ & $<0.001$ \\
\hline Body mass index $\left(\mathrm{kg} / \mathrm{m}^{2}\right)$ & $20.9 \pm 2.3$ & $24.1 \pm 3.6$ & $<0.001$ \\
\hline \multicolumn{4}{|l|}{ Risk factors } \\
\hline Hypertension $(n(\%))$ & $0(0)$ & $46(48)$ & $<0.001$ \\
\hline Dyslipidemia $(n(\%))$ & $0(0)$ & $75(79)$ & $<0.001$ \\
\hline Diabetes mellitus $(n(\%))$ & $0(0)$ & $13(14)$ & 0.018 \\
\hline Current smokers $(n(\%))$ & $0(0)$ & $28(29)$ & $<0.001$ \\
\hline Systolic blood pressure (mm Hg) & $113.2 \pm 11.5$ & $127.2 \pm 13.2$ & $<0.001$ \\
\hline Diastolic blood pressure (mm Hg) & $71.5 \pm 7.2$ & $82.5 \pm 9.4$ & $<0.001$ \\
\hline Brachial arterial FMD (\%) & $12.8 \pm 5.1$ & $7.2 \pm 5.0$ & $<0.001$ \\
\hline BPT (s) & $37.1 \pm 3.8$ & $41.6 \pm 6.7$ & $<0.001$ \\
\hline Hematocrit (\%) & $41.3 \pm 2.6$ & $44.2 \pm 3.5$ & $<0.001$ \\
\hline White blood cell count $\left(\times 10^{3} / \mu \mathrm{L}\right)$ & $4.9 \pm 1.0$ & $5.8 \pm 1.5$ & 0.002 \\
\hline Platelet count $\left(\times 10^{3} / \mu \mathrm{L}\right)$ & $222.2 \pm 47.0$ & $224.3 \pm 43.2$ & 0.812 \\
\hline Fibrinogen (mg/dL) & $246.2 \pm 50.2$ & $273.4 \pm 64.1$ & 0.022 \\
\hline Fasting plasma glucose $(\mathrm{mg} / \mathrm{dL})$ & $91.4 \pm 7.4$ & $101.4 \pm 18.9$ & 0.002 \\
\hline Hemoglobin A1c (\%) & $5.4 \pm 0.3$ & $5.9 \pm 1.0$ & 0.004 \\
\hline Insulin $(\mu \mathrm{U} / \mathrm{mL})$ & $4.6 \pm 2.8$ & $6.8 \pm 5.3$ & 0.017 \\
\hline Total cholesterol (mg/dL) & $190.1 \pm 31.9$ & $215.3 \pm 38.0$ & $<0.001$ \\
\hline HDL cholesterol (mg/dL) & $66.0 \pm 11.6$ & $53.5 \pm 12.7$ & $<0.001$ \\
\hline Triglyceride (mg/dL) & $71.2 \pm 24.5$ & $140.1 \pm 102.6$ & $<0.001$ \\
\hline LDL cholesterol (mg/dL) & $102.6 \pm 24.7$ & $129.7 \pm 34.0$ & $<0.001$ \\
\hline C-reactive protein (mg/dL) & $0.03 \pm 0.03$ & $0.10 \pm 0.20$ & 0.028 \\
\hline Whole blood viscosity (cP) & $3.9 \pm 0.4$ & $4.2 \pm 0.4$ & $<0.001$ \\
\hline
\end{tabular}

Values are presented as means \pm standard deviation. FMD $=$ flow-mediated vasodilation, BPT $=$ blood passage time, $\mathrm{HDL}=$ high-density lipoprotein, $\mathrm{LDL}=$ low-density lipoprotein. 
significantly between the groups. The proportion of males to females, the prevalences of hypertension, dyslipidemia, and diabetes mellitus, and the proportion of current smokers were significantly higher in patients with coronary risk factors than those in healthy controls $($ all $p<0.05)$.

\subsection{Correlation between blood passage time and flow-mediated vasodilatation}

In healthy controls, the correlations between BPT and brachial arterial FMD and other clinical variables are shown in Tables 2 and 3. Univariate regression analysis revealed BPT to be significantly negatively correlated with brachial arterial FMD $(r=-0.325, p<0.05)$ and HDL-C $(r=-0.393$, $p<0.05)$, and positively correlated with BMI $(r=0.530, p<0.01)$ and fibrinogen level $(r=0.335$, $p<0.05)$, but not significantly associated with other clinical variables. Multivariate regression analysis revealed that BPT was significantly positively correlated with BMI $(p<0.01)$ and fibrinogen $(p<0.01)$, but not significantly associated with other clinical variables.

Table 2

Pearson's correlation coefficients to express the correlations between blood passage time and brachial arterial flow-mediated vasodilatation values and other clinical variables in control participants

\begin{tabular}{lrr}
\hline Variable & $r$ & $p$-value \\
\hline Brachial arterial FMD & -0.325 & 0.049 \\
Age & 0.236 & 0.159 \\
Body mass index & 0.530 & 0.001 \\
Systolic blood pressure & 0.206 & 0.221 \\
Diastolic blood pressure & 0.234 & 0.164 \\
Hematocrit & 0.171 & 0.313 \\
White blood count & 0.022 & 0.896 \\
Platelet count & 0.089 & 0.601 \\
Fibrinogen & 0.335 & 0.042 \\
Fasting plasma glucose & -0.074 & 0.665 \\
Hemoglobin A1c & 0.004 & 0.983 \\
Insulin & 0.076 & 0.654 \\
Total cholesterol & -0.048 & 0.779 \\
HDL cholesterol & -0.393 & 0.016 \\
Triglyceride & 0.073 & 0.668 \\
LDL cholesterol & 0.122 & 0.473 \\
C-reactive protein & 0.121 & 0.474 \\
Whole blood viscosity & 0.084 & 0.625 \\
\hline
\end{tabular}

$\mathrm{FMD}=$ flow-mediated vasodilation, $\mathrm{HDL}=$ high-density lipoprotein, $\mathrm{LDL}=$ low-density lipoprotein.

Table 3

Independent predictors of blood passage time identified by multiple regression analysis in control participants

\begin{tabular}{lcc}
\hline Variable & $\beta$ & $p$-value \\
\hline Brachial arterial FMD & 0.001 & 0.993 \\
Body mass index & 0.489 & 0.002 \\
Fibrinogen & 0.434 & 0.005 \\
HDL cholesterol & -0.216 & 0.147
\end{tabular}

FMD = flow-mediated vasodilation, $\mathrm{HDL}=$ high-density lipoprotein. 
In patients with coronary risk factors, the correlations between BPT and brachial arterial FMD and other clinical variables are shown in Fig. 1 and Tables 4 and 5. Univariate regression analysis revealed that BPT was significantly negatively correlated with brachial arterial FMD $(r=-0.331$, $p<0.01)$ and HDL-C $(r=-0.241, p<0.05)$ and significantly positively correlated with BMI $(r=0.290$, $p<0.01)$, hematocrit $(r=0.422, p<0.001)$, WBC count $(r=0.295, p<0.01)$, platelet count $(r=0.204$, $p<0.05)$, and insulin level $(r=0.210, p<0.05)$, but not significantly associated with other clinical variables. Multivariate regression analysis revealed that BPT was significantly negatively correlated with brachial arterial FMD $(p<0.05)$ and significantly positively correlated with hematocrit $(p<0.01)$, but not significantly associated with other clinical variables.

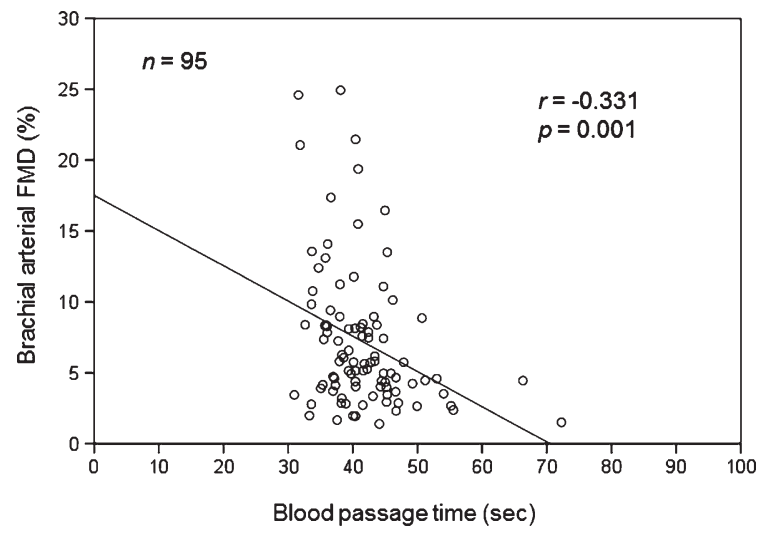

Fig. 1. Correlation between blood passage time and brachial arterial flow-mediated vasodilatation (FMD) in patients with coronary risk factors.

Table 4

Pearson's correlation coefficients expressing the correlations between blood passage time and brachial arterial flow-mediated vasodilatation values and other clinical variables in patients with coronary risk factors

\begin{tabular}{lrr}
\hline Variable & $r$ & $p$-value \\
\hline Brachial arterial FMD & -0.331 & 0.001 \\
Age & 0.041 & 0.697 \\
Body mass index & 0.290 & 0.004 \\
Systolic blood pressure & -0.048 & 0.648 \\
Diastolic blood pressure & -0.043 & 0.676 \\
Hematocrit & 0.422 & $<0.001$ \\
White blood cell count & 0.295 & 0.004 \\
Platelet count & 0.204 & 0.047 \\
Fibrinogen & 0.173 & 0.093 \\
Fasting plasma glucose & 0.062 & 0.554 \\
Hemoglobin A1c & 0.034 & 0.744 \\
Insulin & 0.210 & 0.041 \\
Total cholesterol & -0.085 & 0.413 \\
HDL cholesterol & -0.241 & 0.018 \\
Triglyceride & 0.069 & 0.509 \\
LDL cholesterol & -0.011 & 0.916 \\
C-reactive protein & 0.151 & 0.145 \\
Whole blood viscosity & 0.125 & 0.241 \\
\hline
\end{tabular}

$\mathrm{FMD}=$ flow-mediated vasodilation, $\mathrm{HDL}=$ high-density lipoprotein, $\mathrm{LDL}=$ low-density lipoprotein. 
Table 5

Independent predictors of blood passage time (BPT) identified by multiple regression analysis in patients with coronary risk factors

\begin{tabular}{lcr}
\hline Variable & $\beta$ & $p$-value \\
\hline Brachial arterial FMD & -0.237 & 0.011 \\
Body mass index & 0.119 & 0.260 \\
Hematocrit & 0.306 & 0.003 \\
White blood cell count & 0.145 & 0.143 \\
Platelet count & 0.187 & 0.051 \\
Insulin & -0.009 & 0.929 \\
HDL cholesterol & -0.051 & 0.622 \\
\hline
\end{tabular}

$\mathrm{FMD}=$ flow-mediated vasodilation, $\mathrm{HDL}=$ high-density lipoprotein.

\section{Discussion}

In this study, we showed for the first time that BPT is higher and brachial arterial FMD is lower in patients with coronary risk factors than that in healthy control participants. In healthy controls, there were no significant correlation between BPT and brachial arterial FMD. In patients with coronary risk factors, however, BPT was significantly inversely correlated with brachial arterial FMD.

Several studies [24, 29, 33, 42, 43, 46] have demonstrated impaired blood rheology in patients with coronary risk factors using the microchannel method. In untreated hypertensive patients, BPT and blood pressure were found to be significantly higher than those in normotensive participants [46]. In dyslipidemic patients, BPT was significantly greater than that in normolipidemic individuals $[29,33]$. In men with metabolic syndrome, which includes obesity, dyslipidemia, hypertension, and hyperglycemia, BPT was significantly higher than that in men without metabolic syndrome [24]. Furthermore, in smoking patients, BPT was significantly correlated with smoking variables such as daily tobacco consumption, and smoking cessation resulted in a markedly decreased BPT 3 months after the start of therapy [43]. In contrast, several studies [5, 7, 13, 14] have reported endothelial dysfunction in patients with coronary risk factors based on the assessment of brachial arterial FMD. Brachial arterial FMD was significantly less in essential hypertensive [14], hypercholesterolemic [5], and noninsulindependent diabetic patients [13] than that in healthy controls. The effect of cigarette smoking was dose-related, and smokers exhibited impaired brachial arterial FMD relative to healthy young adults [7]. Rossi et al. [39] also have demonstrated that peripheral microvascular dysfunction, assessed by forearm skin post-occlusive reactive hyperemia (PORH) test, may contribute to atherosclerotic damage in type 1 diabetes patients. This skin PORH test may be considered a measure of skin vasoreactivity, mostly dependent from the myogenic function of the microvascular wall and only, to a lesser extent, from the microvascular endothelial function. These findings support the results of our study. Thus, the presence of these factors may negatively influence blood rheology and endothelial function in patients with coronary risk factors.

Few studies demonstrate an association between blood rheology and brachial arterial endothelial function in patients with coronary risk factors. However, an association between blood rheology as assessed by MC-FAN and other surrogate markers of atherosclerosis, such as pulse-wave velocity (PWV) and carotid intima-medial thickness (IMT), has been shown in patients with coronary risk factors in a few studies [21, 41]. PWV and carotid IMT are also widely used to assess functional and structural vascular damage. Satoh et al. [41] reported that BPT was closely positively correlated with PWV in obese patients, suggesting that impaired blood rheology may be closely associated with increased arterial stiffness in these patients. Kobayashi et al. [21] demonstrated that BPT was 
significantly well correlated with PWV and carotid IMT in patients with hemodialysis, suggesting that blood rheology may contribute to atherosclerosis in uremic patients undergoing hemodialysis. This study also revealed that BPT is negatively associated with brachial arterial endothelial function (as measured by FMD) in patients with coronary risk factors. Although there were differences in the methods used to assess clinical atherosclerosis between our and these two studies, their findings are consistent with the results of this study. Accordingly, it is likely that a negative association is present between blood rheology and brachial arterial endothelial function in patients with coronary risk factors.

The reasons why blood rheology (as measured by MC-FAN) is negatively associated with endothelial function (as measured by brachial arterial FMD) in patients with coronary risk factors but not in healthy controls remain unclear. However, there are several possible explanations. Blood rheology may interact with endothelial function; an impaired FMD response reflects endothelial dysfunction. Because FMD is predominantly dependent on endothelium-derived nitric oxide (NO) [15, 31], impaired FMD is characterized by a decreased production and/or local bioavailability of NO. NO increases RBC and platelet deformability, and reduces platelet aggregation and leukocyte adhesion [6, 20, 48]. Reportedly, RBC deformability, leucocyte adhesiveness, and platelet aggregation also affect blood rheology measured using the microchannel method [19, 29, 32, 33, 42, 43, 45, 46]. Therefore, endothelial dysfunction measured by brachial arterial FMD may be associated with impaired blood rheology as measured by MC-FAN in patients with coronary risk factors. In contrast, elevated RBC aggregation and impaired RBC deformability, which are related to impaired blood rheology as measured using the microchannel method [19, 29, 32, 33, 42, 43, 45, 46], suppress the expression and activity of NO synthase [2]. In a rat model, Baskurt et al. [2] demonstrated that enhanced RBC aggregation results in suppressed expression of NO synthesizing mechanisms, leading to altered vasomotor tonus; the mechanisms involved are most likely related to decreased wall shear stresses due to decreased blood flow and/or increased axial accumulation of RBCs. Furthermore, Naruse et al. [34] reported that long-term administration of N omega-nitro-L-arginine methylester (L-NAME), an inhibitor of NO synthase, causes further impairment of endothelium-dependent relaxation in the hypercholesterolemic rabbit thoracic aorta. Thus, impaired blood rheology as measured by MC-FAN may be associated with endothelial dysfunction as measured by brachial arterial FMD in patients with coronary risk factors.

Forconi et al. [10, 11] and Intaglietta et al. [40] have reported the association between hemorheology and endothelial function. They have hypothesized that increased blood viscosity is associated with endothelial activation. In the present study, while whole blood viscosity levels were significantly greater in patients with coronary risk factors than those in healthy controls, there were no significant associations between whole blood viscosity and FMD in the healthy control participants $(r=-0.242$, $p=0.154$ ) (data not shown) as well as in the patients with coronary risk factors $(r=-0.038, p=0.726)$ (data not shown). Parkhust et al. [36] also have reported that whole blood viscosity is not significantly correlated with FMD in healthy populations without any overt disease. This finding supports the results of the present study. Since the present study does not include severe diseased populations, the lack of significant associations between whole blood viscosity and FMD may be obtained by a relative narrow range of whole blood viscosity values in our sample ranging from 3.17 to $5.10 \mathrm{cP}$. Thus, it is possible that the present study showed no associations between whole blood viscosity and FMD.

This study has several limitations. First, the number of participants enrolled was relatively small. A larger number of participants are required. Second, the study may have been subject to bias because the study participants ranged from young to elderly patients with or without various coronary risk factors. A cohort that was homogeneous in terms of age and health status is required. Third, in this study, a proportion of patients with coronary risk factors were receiving medications such as antihypertensive, lipid-lowering, and hypoglycemic agents, which may complicate the interpretation of the results. Finally, because the results range for healthy control participants in this study was small, it is possible that there was no significant association between BPT and brachial arterial FMD in healthy controls. 


\section{Conclusions}

In this study, we demonstrated that patients with coronary risk factors have impaired blood rheology associated with decreased flow-mediated vasodilation. Our data suggest that measurement of blood rheology using the microchannel method may be useful in evaluating flow-mediated vasodilation as a marker of atherosclerosis in patients with coronary risk factors. Further studies are needed to clarify the relationship between blood rheology and flow-mediated vasodilation in patients with coronary risk factors as well as in healthy individuals.

\section{Acknowledgments}

This study was financially supported in part by a grant-in-aid for Scientific Research I (No. 23590661 and No. 26460666) from the Ministry of Education, Culture, Sports, Science and Technology of Japan. We are grateful to Mayumi Nishiyama, Reiko Shizuka, Shigeki Yabe, Masaki Hayakawa, Yoshio Takahashi, Nobuo Kotajima, Kiyomi Nakajima, Toshiya Inoue, Chiaki Suto, Sakie Fujii, and Tetsuo Machida for technical assistance.

\section{References}

[1] American Diabetes Association, Diagnosis and classification of diabetes mellitus, Diabetes Care 35 (2012), S64-S71.

[2] O.K. Baskurt, O. Yalcin, S. Ozdem, J.K. Armstrong and H.J. Meiselman, Modulation of endothelial nitric oxide synthase expression by red blood cell aggregation, Am J Physiol Heart Circ Physiol 286 (2004), H222-H229.

[3] D. Behrendt and P. Ganz, Endothelial function. From vascular biology to clinical applications, Am J Cardiol 90 (2002), 40L-48L.

[4] M. Bor-Kucukatay, R.B. Wenby, H.J. Meiselman and O.K. Baskurt, Effects of nitric oxide on red blood cell deformability, Am J Physiol Heart Circ Physiol 284 (2003), H1577-H1584.

[5] P.R. Casino, C.M. Kilcoyne, A.A. Quyyumi, J.H. Hoeg and J.A. Panza, The role of nitric oxide in endothelium-dependent vasodilation of hypercholesterolemic patients, Circulation 88 (1993), 2541-2547.

[6] W.P. Castelli, Epidemiology of coronary heart disease: The Framingham study, Am J Med 76 (1984), 4-12.

[7] D.S. Celermajer, K.E. Sorensen, D. Georgakopoulos, C. Bull, O. Thomas, J. Robinson and J.E. Deanfield, Cigarette smoking is associated with dose-related and potentially reversible impairment of endothelium-dependent dilation in healthy young adults, Circulation 88 (1993), 2149-2155.

[8] D.S. Celermajer, K.E. Sorensen, V.M. Gooch, D.J. Spiegelhalter, O.I. Miller, I.D. Sullivan, J.K. Lloyd and J.E. Deanfield, Non-invasive detection of endothelial dysfunction in children and adults at risk of atherosclerosis, Lancet 340 (1992), 1111-1115.

[9] E. Ernst and K.L. Resch, Fibrinogen as a cardiovascular risk factor: A meta-analysis and review of the literature, Ann Intern Med 118 (1993), 956-963.

[10] S. Forconi and T. Gori, Endothelium and hemorheology, Clin Hemorheol Microcirc 53 (2013), 3-10.

[11] S. Forconi and T. Gori, The evolution of the meaning of blood hyperviscosity in cardiovascular physiopathology: Should we reinterpret Poiseuille? Clin Hemorheol Microcirc 42 (2009), 1-6.

[12] G. Galea and R.J.L. Davidson, Haematological and haemorheological changes associated with cigarette smoking, J Clin Pathol 38 (1985), 978-984.

[13] J. Goodfellow, M.W. Ramsey, L.A. Luddington, C.J. Jones, P.A. Coates, F. Dunstan, M.J. Lewis, D.R. Owens and A.H. Henderson, Endothelium and inelastic arteries: An early marker of vascular dysfunction in non-insulin dependent diabetes, BMJ 312 (1996), 744-745.

[14] K. Iiyama, M. Nagano, Y. Yo, N. Nagano, K. Kamide, J. Higaki, H. Mikami and T. Ogihara, Impaired endothelial function with essential hypertension assessed by ultrasonography, Am Heart J 132 (1996), 779-782.

[15] R. Joannides, W.E. Haefeli, L. Linder, V. Richard, E.H. Bakkali, C. Thuillez and T.F. Lüscher, Nitric oxide is responsible for flow-dependent dilatation of human peripheral conduit arteries in vivo, Circulation 91 (1995), 1314-1319.

[16] W.B. Kannel, W.P. Castelli, T. Gordon and P.M. McNamara, Serum cholesterol, lipoproteins, and the risk of coronary heart disease. The Framingham study, Ann Intern Med 74 (1971), 1-12. 
[17] W.B. Kannel, T.R. Dawber, A. Kagan, N. Revotski and J. Stokes 3rd., Factors of risk in the development of coronary heart disease-six year follow-up experience. The Framingham study, Ann Intern Med 55 (1961), 33-50.

[18] W.B. Kannel and D.L. McGee, Diabetes and cardiovascular disease. The Framingham study, JAMA 241 (1979), 2035-2038.

[19] Y. Kikuchi, K. Sato, H. Ohki and T. Kaneko, Optically accessible microchannels formed in a single-crystal silicon substrate for studies of blood rheology, Microvasc Res 44 (1992), 226-240.

[20] P. Kleinbongard, R. Schulz, T. Rassaf, T. Lauer, A. Dejam, T. Jax, I. Kumara, et al., Red blood cells express a functional endothelial nitric oxide synthase, Blood 107 (2006), 2943-2951.

[21] S. Kobayashi, K. Okamoto, K. Maesato, H. Moriya and T. Ohtake, Important role of blood rheology in atherosclerosis of patients with hemodialysis, Hemodial Int 9 (2005), 268-274.

[22] W. Koenig, M. Sund, E. Ernst, W. Mraz, V. Hombach and U. Keil, Association between rheology and components of lipoproteins in human blood. Results from the MONICA Project, Circulation 85 (1992), 2197-2204.

[23] W. Koenig, M. Sund, B. Filipiak, A. Döring, H. Löwel and E. Ernst, Plasma viscosity and the risk of coronary heart disease: Results from the MONICA-Augsburg Cohort Study, 1984 to 1992, Arterioscl Thromb Vasc Biol 18 (1998), 768-772.

[24] K. Kotani, A. Inata and S. Araga, Hemorheology by microchannel method in males with metabolic syndrome, Arch Med Res 38 (2007), 463-464.

[25] A.J. Lee, The role of rheological and haemostatic factors in hypertension, J Hum Hypertens 11 (1997), 767-776.

[26] C.Y. Lee, K.C. Kim, H.W. Park, J.H. Song and C.H. Lee, Rheological properties of erythrocytes from male hypercholesterolemia, Microvasc Res 67 (2004), 133-138.

[27] G.Y. Lip and D.G. Beevers, Abnormalities of rheology and coagulation in hypertension, J Hum Hypertens 8 (1994), 693-702.

[28] G.D. Lowe, A.J. Lee, A. Rumley, J.F. Price and F.G. Fowkes, Blood viscosity and risk of cardiovascular events: The Edinburgh Artery Study, Br J Haematol 96 (1997), 168-173.

[29] T. Machida, H. Sumino, M. Fukushima, N. Kotajima, H. Amagai and M. Murakami, Blood rheology and the low-density lipoprotein cholesterol/high-density lipoprotein cholesterol ratio bin dyslipidaemic and normolipidaemic subjects, $J$ Int Med Res 38 (2010), 1975-1984.

[30] Y. Maki, Y. Endo, M. Fukushima, M. Nishiyama, T. Dobashi, H. Sumino and M. Murakami, Estimation of viscosity from passage time of liquids flowing through a microchannel array, J Biorheol 26 (2013), 69-73.

[31] I.T. Meredith, K.E. Currie, T.J. Anderson, M.A. Roddy, P. Ganz and M.A. Creager, Postischemic vasodilation in human forearm is dependent on endothelium-derived nitric oxide, Am J Physiol 270 (1996), H1435-H1440.

[32] Y. Muranaka, F. Kunimoto, J. Takita, H. Sumino, M. Nara, H. Kuwano and M. Murakami, Impaired blood rheology in critically ill patients in an intensive care unit, J Int Med Res 34 (2006), 419-427.

[33] M. Nara, H. Sumino, M. Nara, T. Machida, H. Amagai, K. Nakajima and M. Murakami, Impaired blood rheology and elevated remnant-like lipoprotein particle cholesterol in hypercholesterolaemic subjects, J Int Med Res 37 (2009), 308-317.

[34] K. Naruse, K. Shimizu, M. Muramatsu, Y. Toki, Y. Miyazaki, K. Okumura, H. Hashimoto and T. Ito, Long-term inhibition of NO synthesis promotes atherosclerosis in the hypercholesterolemic rabbit thoracic aorta. PGH2 does not contribute to impaired endothelium-dependent relaxation, Arterioscler Thromb 14 (1994), 746-752.

[35] T. Ogihara, K. Kikuchi, H. Matsuoka, T. Fujita, J. Higaki, M. Horiuchi, Y. Imai, et al., The Japanese Society of Hypertension Guidelines for the Management of Hypertension (JSH 2009), Hypertens Res 32 (2009), 3-107.

[36] K.L. Parkhurst, H.F. Lin, A.E. Devan, J.N. Barnes, T. Tarumi and H. Tanaka, Contribution of blood viscosity in the assessment of flow-mediated dilation and arterial stiffness, Vasc Med 17 (2012), 231-234.

[37] O.T. Raitakari and D.S. Celermajer, Flow-mediated dilatation, Br J Clin Pharmacol 50 (2000), $397-404$.

[38] R. Ross, Atherosclerosis: An inflammatory disease, N Engl J Med 340 (1999), 115-126.

[39] M. Rossi, E. Matteucci, M. Pesce, C. Consani, F. Franzoni, G. Santoro and O. Giampietro, Peripheral microvascular dysfunction as an independent predictor of atherosclerotic damage in type 1 diabetes patients: A preliminary study, Clin Hemorheol Microcirc 54 (2013), 381-391.

[40] B.Y. Salazar Vázquez, J. Martini, A. Chávez Negrete, A.G. Tsai, S. Forconi, P. Cabrales, P.C. Johnson and M. Intaglietta, Cardiovascular benefits in moderate increases of blood and plasma viscosity surpass those associated with lowering viscosity: Experimental and clinical evidence, Clin Hemorheol Microcirc 44 (2010), 75-85.

[41] N. Satoh, K. Kotani, H. Wada, A. Himeno, S. Shimada, Y. Sasaki, K. Yamada, A. Shimatsu and K. Hasegawa, Unfavorable blood rheology is closely associated with arterial stiffness in obese patients, Endocr J 56 (2009), 915-918.

[42] K. Seki, H. Sumino, M. Nara, N. Ishiyama, M. Nishino and M. Murakami, Relationships between blood rheology and age, body mass index, blood cell count, fibrinogen, and lipids in healthy subjects, Clin Hemorheol Microcirc 34 (2006), 401-410. 
[43] S. Shimada, K. Hasegawa, H. Wada, S. Terashima, N. Satoh-Asahara, H. Yamakage, S. Kitaoka, M. Akao, A. Shimatsu and Y. Takahashi, High blood viscosity is closely associated with cigarette smoking and markedly reduced by smoking cessation, Circ J 75 (2011), 185-189.

[44] H. Sumino, S. Ichikawa, Y. Ohyama, T. Takahashi, Y. Saito, T. Nakamura, T. Kanda and M. Kurabayashi, Effect of transdermal hormone replacement therapy on the monocyte chemoattractant protein-1 concentrations and other vascular inflammatory markers and on endothelial function in postmenopausal women, Am J Cardiol 96 (2005), $148-153$.

[45] H. Sumino, S. Ichikawa, T. Takahashi, H. Sakamoto, K. Goto-Onozato, S. Koya, T. Kanda, M. Nara, K. Seki, M. Murakami and M. Kurabayashi, Conjugated estrogen plus medroxyprogesterone does not impair blood rheological properties in hypertensive postmenopausal women, Maturitas 53 (2005), 306-314.

[46] H. Sumino, M. Nara, K. Seki, T. Takahashi, T. Kanda, S. Ichikawa, K. Goto-Onozato, S. Koya, M. Murakami and M. Kurabayashi, Effect of antihypertensive therapy on blood rheology in patients with essential hypertension, $J$ Int Med $\operatorname{Res} 33$ (2005), 170-177.

[47] T. Teramoto, J. Sasaki, S. Ishibashi, S. Birou, H. Daida, S. Dohi, G. Egusa, et al., Executive summary of the Japan Atherosclerosis Society (JAS) guidelines for the diagnosis and prevention of atherosclerotic cardiovascular diseases in Japan -2012 version, J Atheroscler Thromb 20 (2013), 517-523.

[48] J.R. Vane, E.E. Anggard and R.M. Botting, Regulatory functions of the vascular endothelium, N Engl J Med 323 (1990), 27-36.

[49] P.A. Wolf, R.B. D'Agostino, W.B. Kannel, R. Bonita and A.J. Belanger, Cigarette smoking as a risk factor for stroke. The Framingham Study, JAMA 259 (1988), 1025-1029. 